\title{
Contract design for the fourth party logistics considering tardiness risk
}

\author{
Hongyan Wanga,d, Min Huanga,d, Hongfeng Wang ${ }^{\mathrm{a}}$, Xuehao Feng ${ }^{\mathrm{b}}$ and Yanjie Zhou ${ }^{\mathrm{c}^{*}}$
}

${ }^{a}$ College of Information Science and Engineering, Northeastern University, Shenyang 110819, China

${ }^{b}$ Ocean College, Zhejiang University, Zhoushan 316021, China

${ }^{c}$ School of Management Engineering, Zhengzhou University, Zhengzhou 450001, China

${ }^{d}$ State Key Laboratory of Synthetical Automation for Process Industries, Northeastern University, Shenyang 110819, China

\section{H R O N I C L E \\ A B S T R A C T}

Article history:

Received August 222021

Received in Revised Format

August 252021

Accepted September 152021

Available online

September, 152021

Keywords:

Fourth party logistics

Contract design

Risk management

Tardiness risk index
Nowadays, tardiness has become a significant risk in the logistics industry. To address this problem, we introduce the tardiness risk index to quantify both the magnitude of the tardiness risk and the maximum probability of tardiness occurring. In this paper, we investigate the contract design problem with the tardiness risk index to mitigate the tardiness risk when a fourth-party logistics company (4PL) delegates the delivery task of a client to a third-party logistics company (3PL). Specifically, the contracts are designed in a decentralized system with information symmetry and information asymmetry when 3PL is risk neutral and risk averse. Furthermore, the incentive problems demonstrated that the 3PL is encouraged to make the optimal effort for delivery and the 4PL determines the optimal fixed payment and penalty coefficient. Through analyzing the experimental simulation results, we can find that the contract can effectively mitigate the tardiness risk and the maximum probability of risk occurrence.

\section{Introduction}

The huge logistics business brings opportunities as well as challenges to the logistics industry. With the development of logistics, the client expects faster and more flexible logistics services. However, the traditional logistics relying on the 3PL which does not integrate logistics resources cannot meet the client's requirements of faster delivery. The 4PL, as a logistics integrator with an integrated operation model, can obtain higher delivery efficiency. For example, "Cainiao", a logistics affiliate of China Alibaba Group, integrates existing industry logistics resources and connects carriers and shippers to implement efficient transportation solutions (Cainiao Network Overview, 2016). Specifically, Cainiao provides the client with comprehensive logistics service solutions based on the platform, while the 3PL on it performs the actual logistics operation and delivery. However, based on the client feedback from the Cainiao platform, the 3PL may not deliver the goods on time and experience tardiness. In addition, according to a report released by the PRC State Post Bureau in December 2018, tardiness is the major issue of client complaints about express delivery services, which accounts for $31.7 \backslash \%$ of the total valid appeals (PRC State Post Bureau, 2018). Therefore, it is crucial to reduce the tardiness risk. There are some studies about time reduction for the manufacturing systems (Yamada et al., 2021; Çetinkaya et al., 2021), however, as an important service criterion for evaluating the delivery performance, few studies have focused on tardiness risk management in the design of logistics service supply chain contract (Heckmann et al., 2015).

There are two major potential threats for the occurrence of tardiness. The first threats belong to external factors, including weather conditions, service road conditions, and government policies. The second threats belong to internal factors, including backward equipment and wrong delivery due to employee negligence. The 3PL can improve logistics operations efficiency if they invest in advanced equipment or a skilled workforce, etc. (Huang et al., 2019).

* Corresponding author

E-mail: ieyjzhou@zzu.edu.cn (Y. Zhou)

2022 Growing Science Ltd. doi: $10.5267 /$ j.ijiec.2021.9.002 
However, since the 3PL pays for the actual delivery and may have no incentive to invest or improve their delivery process, if the 4PL does not go to incentivize the 3PL and pays a mere fee regardless of the delivery performance. Moreover, risk management concerns not only the magnitude of the risk but also the probability of the risk occurrence. Hence the main objective of this paper is to design contracts to mitigate the tardiness risk and the probability of the risk occurrence, and focuses on solving the following questions:

(1) How does the 4PL design contracts to motivate 3PL to make more efforts to mitigate the tardiness risk and the risk occurrence probability?

(2) How does 3PL's asymmetric cost information impact the tardiness risk?

(3) How does 3PL's degree of risk aversion affect the tardiness risk?

To measure the magnitude of the tardiness risk, we adopt a tardiness risk index. The advantage of the tardiness risk index is that it not only measures the magnitude of the tardiness risk but also quantifies the maximum probability of violation occurrence (Jaillet et al, 2016; Adulyasak and Jaillet, 2015). The index is from Jaillet et al. (2016) who proposed the performance index to quantify the risk of requirement violations for an uncertain attribute in a routing problem. In addition, to motivate the 3PL to mitigate the tardiness risk, we endogenize the actual delivery time, and let it relates to 3PL's effort level, i.e., the 3PL can take action to reduce the tardiness risk (Huang et al., 2016). However, the 3PL's effort level is unobservable to the 4PL and difficult to be monitored. Therefore, as the principal, the 4PL usually inevitably uses contractual incentives to induce the 3PL to make more efforts (Laffont \& Martimort, 2002; Zhou \& Kim, 2019). We first establish a centralized system to minimize the system's tardiness risk. Second, as the 4PL and 3PL have different objectives and make decisions separately, using the principal-agent theory, we establish the model considering the tardiness risk index in the decentralized system. Specifically, as the cost coefficient of 3PL is unobservable to the 4PL, we assume that the 3PL may be a high type with a lower cost coefficient or a low type with a higher cost coefficient. Also, because the 3PL performs the actual delivery and invests in delivery, we assume that the 3PL is risk averse, while the 4PL is risk neutral considering that she does not pay the 3PL until the delivery is complete (Huang et al., 2019). Thus, we establish the decentralized models when the 3PL is risk-neutral and risk-averse under symmetric and asymmetric cost information, respectively. The problem under the asymmetric information is both a moral hazard problem (3PL's unobservable effort level) and an adverse selection problem (asymmetric cost coefficient information).

We find that the tardiness risk and 3PL's effort level in the decentralized system under symmetric information (hereafter referred to as symmetric scenario) are equal to that in the centralized system. Thus, delegation is costless for the 4PL under complete information, and the 4PL achieves the same risk level that she would get if she is carrying out the task herself (with the same cost function as the 3PL). The effort level of the high type 3PL in the decentralized system under asymmetric information (hereafter referred to as asymmetric scenario) is smaller than that in the centralized system, and the effort level of the low type 3PL is equal in both cases, then the tardiness risk is larger in the asymmetric scenario that in the centralized system. This is because the high type 3PL in the asymmetric scenario gets the information rent by mimicking the low type 3PL. In the asymmetric scenario, the numerical experiments show that comparing the tardiness risk when 3PL is risk neutral with that he is risk averse, it is found that the tardiness risk increases in the degree of the risk aversion.

\section{Literature review}

As a part of supply chain risk management (Govindan \& Chaudhuri, 2016; Choi et al., 2016), risk management in the logistics service supply chain has been studied by some researchers from the perspective of quality risks and demand risks. Quality risks in logistics may include damage or loss of goods. Huang et al. (2019) designed optimal contracts for 4PL to make the 3PL ensure freight quality. Liu and Wang (2015) studied the optimal mix of risk attitudes for quality control, and the results show that a logistics service integrator is more inclined to choose risk-seeking logistics service providers. Furthermore, some studies focus on demand uncertainty in the logistics service supply chain. Liu et al. (2015) focused on research on service quality guarantee problems with demand updating in the logistics service supply chain. Zhang et al. (2017) studied the problem of collaborative decision-making by carriers with uncertain demand in e-commerce logistics networks. The second stream relates to contract issues in the logistics service supply chain. Specifically, some researchers examined the single contract design issues. Lim (2000) established a contract in order that the third-party logistics service providers can reveal their true capabilities to the logistics buyer, and used the revelation principle to develop the game-theoretic model. Zhang et al. (2019) studied two logistics service providers among different competitive power structures with horizontal collaboration, and designed a revenue sharing contract to empower coordination in the E-commerce logistics. Ogier et al. (2013) studied multiperiod planning consisting of productions, transportations, and storage activities, using the quantity discount contract to coordinate the decentralized system. Some researchers investigate the performance of two or more different contracts. Gong et al. (2018) analysed the impact of four different contract structures on the level of investment of 3PL. Wang et al. (2018) studied two contracts to examine the impact of shipment insurance premium. When space requirements are random, Chen et al. (2001) considered space commitments that can be modified and study three forms of contracts between the user and thirdparty warehouse. Alp et al. (2003) examined the design of transportation contracts by a manufacturer with a transportation company, and presented a method to design transportation contracts. Liu et al. (2018) took into account the characteristics of 
mass customization in the OBOR region, exploring and evaluating cost-sharing contracts under four structures to improve logistics services in the region.

In addition, other researchers examine how to design contracts to eliminate the double marginalization and to induce the logistics service supply chain to act in a coordinated way. Cai et al. (2013) proposed a wholesale-market clearance contract and a wholesale-price-discount sharing contract to coordinate a three-tier supply chain when the product is perishable. Wu et al. (2015) investigated two coordination mechanisms for the decentralized channel between the distributor and the third-party logistics under different power structures. Ogier et al. (2015) studied order allocation problem in decentralized planning in multi-period using minimal-information sharing contracts to coordinate. Recently, Moon et al. (2020) introduced a target rebate contract for sales promotion by coordinating a pair of retailers. The differences between our study and the above are as follows. First, Huang et al. (2019) studied delivery quality risk management in logistics, different from them, our problem is aimed at the 4PL platform tardiness risk management in the logistics service supply chain. We design time-based incentive contracts to minimize the tardiness risk, and endogenize the delivery time by modelling it related to the 3PL's effort level and affected by the external factors. Second, we use a tardiness risk index to measure the risk in logistics risk management, which not only mitigates the magnitude of the tardiness risk, but also the maximum probability of the tardiness risk occurrence. This is different from other risk management papers, which only examine the level of risk mitigation.

\section{Problem model}

Supposing a 4PL platform receives a logistics delivery task required by a client, after which the 4PL delegates the transportation of cargoes to a 3PL. The delivery time of these cargoes is negatively correlated to 3PL's effort level and is uncontrollable for the 4PL. There exist many external random factors that affect the delivery time. Usually, the 3PL's effort level is difficult for the 4PL to predict, and only when the cargoes are received the 4PL can assess the 3PL's effort level by observing the final delivery time. In this paper, we introduce two contract terms including a penalty coefficient and a fixed payment, and a tardiness risk index into the contract, to motivate the 3PL for improving his effort level and mitigating the tardiness risk. The sequence of events is: (i) the 4PL offers a contract considering tardiness risk; (ii) the 3PL decides whether or not to accept the contract, and if accepts, decides the effort level; (iii) the delivery is completed, and the transfer payment is achieved.

\subsection{Notations}

We model the actual delivery time $\tilde{t}$ as a nonnegative random variable, $\tilde{t}=a-b e+\varepsilon$. The regular delivery time $a$ represents the expected delivery time of 3PL with zero efforts, $e$ is 3PL's effort level, and effort output level $b$ represents the output capacity of 3PL, $\varepsilon$ denotes external factors and follows a uniform distribution, $\varepsilon \sim U(-m, m), m \geq 0$. The uniform distribution exhibits the increasing generalized failure rate property (Banciu and Mirchandani, 2013). The 3PL's cost is subject to the commonly used quadratic cost function $k e^{2}$, where $k>0$ denotes the effort cost coefficient and measures how costly it is for the 3PL to deliver goods. A smaller $k$ means that the 3PL is more cost-efficient during the logistics delivery process. We assume that there are two types of 3PL: the high type with lower cost coefficient $k_{h}$ or low type with higher cost coefficient $k_{l}\left(k_{h}<k_{l}\right)$, with the respective probability of $v(0<v<1)$ and $1-v$. The 4PL will receive a payment $B$ from the client about a certain delivery task. Assuming the client's requirement delivery lead time is $\tau$, if the tardiness occurs, the 4PL suffers a penalty of $\rho[\tilde{t}-\tau]^{+}$from the client, $\rho$ is per unit time penalty (i.e., the penalty for each unit of time). Similarly, the penalty imposed on 3PL by 4PL is $\beta \rho[\tilde{t}-\tau]^{+}, \beta$ denotes the penalty coefficient. Besides, the 4PL gives the 3PL a fixed payment $\alpha$. The 4PL provides a contract $(\alpha, \beta)$ for the 3PL. Then at the end of delivery, the 3PL gets payoff $\alpha-\beta \rho[\tilde{t}-\tau]^{+}$. The model notations and descriptions are shown in Table 1.

Table 1

Notations

\begin{tabular}{cl}
\hline Symbols & \multicolumn{1}{c}{ Descriptions } \\
\hline$k$ & 3PL's cost coefficient \\
$a$ & A random variable follows uniform distribution $U(-m, m), m \geq 0$ \\
$b$ & The expected delivery time of the 3PL with zero efforts \\
$\tau$ & 3PL's effort output coefficient \\
$\rho$ & The delivery lead time required by the client \\
$\delta$ & Unit time penalty \\
$\psi$ & 3PL's degree of risk aversion \\
$r$ & Tardiness risk index \\
$B$ & Risk tolerance parameter, $r>0$ \\
\hline Decision variables & The payment that the 4PL receives from the client \\
\hline$\alpha$ & The fixed payment \\
$e$ & The penalty coefficient imposed by the 4PL on the 3PL, $\beta \geq 0$ \\
\hline
\end{tabular}




\section{Derived variables}

$\pi^{c}, \pi^{f}, \pi^{t}$

$\Pi^{c}, \Pi^{f}, \Pi^{t}$

The profits of the centralized system, 4PL and 3PL

The expected utilities of the centralized system, 4PL and 3PL

We assume that the 3PL is risk averse given that he invests in delivery, while the 4PL is risk neutral considering that she does not pay the 3PL until the delivery is complete (Huang et al., 2019). To obtain an analytical solution and better analyze the problem, we will first consider the case that the 3PL is risk neutral in the decentralized system, and then further consider the case that the 3PL is risk averse. We assume that the tardiness risk occurs when the 3PL does not make efforts, i.e., $a+m>$ $\tau$. To ensure that the delivery time is positive, i.e., $a-b e-m>0$ always holds, we assume that $B \leq(4 k m(a-m)-$ $\left.\rho b^{2}(2 m-\tau)\right)^{2} /\left(4 b^{2} m\left(\rho b^{2}+4 k m\right)+\rho k(a+m-\tau)^{2} /\left(\rho b^{2}+4 k m\right)\right.$ and $4 k m(a-m) \geq \rho b^{2}(2 m-\tau)$. We normalize the reservation utility to 0 for both 3PL and 4PL. To make the results more concise, we let $\bar{t}=a+m-\tau$. The 3PL decides his effort level according to the contract to maximize his utility. The model for the logistics service supply chain is shown in Fig. 1.

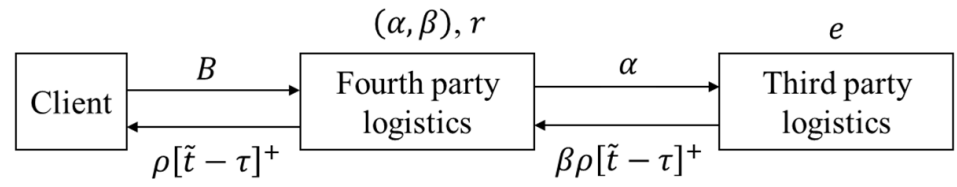

Fig. 1. The model of the logistics service supply chain.

\subsection{Tardiness risk index}

We adopt a risk measure introduced by Jaillet et al. (2016), which can not only measure the magnitude of the tardiness risk, but also provide the maximum probability of tardiness risk occurrence. Given the delivery lead time $\tau$, the tardiness risk index $\psi$ is

$$
\begin{aligned}
& \psi=\min \left\{r \geq 0 \mid C_{r}(\tilde{t}) \leq \tau\right\} \\
& C_{r}(\tilde{t})=r \ln E(\exp (\tilde{t} / r)),
\end{aligned}
$$

where $C_{r}(\tilde{t})$ is the certainty equivalent under exponential disutility $u(\tilde{t})=\exp (\tilde{t} / r), u(\tilde{t})$ represents the 4PL exhibiting a risk aversion behaviour toward the delivery time. The propose of the index was inspired by the study of Aumann and Serrano (2008), that less risk-averse individuals accept riskier gambles in economics, then the riskiness of a gamble is the individual risk tolerance parameter. When the tardiness risk index $\psi$ is given, the probability of the lead time $\tau$ plus any period $\theta$ is up bounded by $\exp (-\theta / \psi)$

$$
p(\tilde{t} \geq \tau+\theta) \leq \exp (-\theta / \psi), \forall \theta \geq 0 \text {. }
$$

The probability $\exp (-\theta / \psi)$ is for the random event that the tardiness exceeds a duration $\theta$, and provides upper bounds of the probability of excess a duration. The upper bounds are available for a series of duration $\theta$, rather than for a single value. Also, a smaller $\psi$ corresponds to a smaller upper bound for the probability. Therefore, in this paper we adopt the method proposed by Jaillet et al. (2016) to allow the manager to reduce the upper bounds of the probability of risk occurrence while reducing the tardiness risk simultaneously. The function $C_{r}(\tilde{t})$ can be derived with the moment generating function. Lemma 1 shows the expression of $C_{r}(\tilde{t})$ that proposed by Jaillet et al. (2016).

Lemma 1. When $\tilde{t}$ follows a uniform distribution $U(-m, m)$, the certainty equivalent of $\tilde{t}$ is

$C_{r}(\tilde{t})=a-b e-m+r \ln \frac{\exp (2 m / r)-1}{2 m / r}$,

where $C_{r}(\tilde{t})$ is monotonically decreasing in $r$.

Proof: See appendix $B$.

\subsection{Centralized system}

In the centralized system, the transfer payment between the 4PL and the 3PL is omitted. The system's objective is to minimize the tardiness risk. The centralized system's profit is

$$
\pi^{c}(e)=B-\rho[\tilde{t}-\tau]^{+}-k e^{2} .
$$

Suppose the centralized system is risk-neutral, then the centralized system's expected utility can be expressed as follows:

$$
\Pi^{c}(e)=B-\rho E\left([\tilde{t}-\tau]^{+}\right)-k e^{2} .
$$


By replacing $E\left([\tilde{t}-\tau]^{+}\right)=\frac{(\tau+b e-a-m)^{2}}{4 m}$, the above equation (5) can be expressed as Eq. (6). The detail derivation of $E\left([\tilde{t}-\tau]^{+}\right)$shows in appendix A.

$$
\Pi^{c}(e)=B-\frac{\rho(a-b e+m-\tau)^{2}}{4 m}-k e^{2}
$$

The system determines the optimal effort level $e$ to minimize the tardiness risk index, we have

$$
\begin{aligned}
& \min _{e} r, \\
& \text { subject to } \\
& C_{r}(\tilde{t}) \leq \tau \\
& \Pi^{c}(e) \geq 0 \\
& e \geq 0, r>0 .
\end{aligned}
$$

Constraint (8) represents that the certainty equivalent of delivery time should be less than the delivery lead time $\tau$ which is required by the client. Constraint (9) ensures that the system earns at least its reservation utility. Solving the problem, we get the following results.

Proposition 1 In the centralized logistics system, if $\frac{\rho k \bar{t}^{2}}{\rho b^{2}+4 k m} \leq B \leq \frac{\left(4 k m(a-m)-\rho b^{2}(2 m-\tau)\right)^{2}}{4 b^{2} m\left(\rho b^{2}+4 k m\right)}+\frac{\rho k \bar{t}^{2}}{\rho b^{2}+4 k m}$, there exist the optimal $\left(e^{c *}, r^{c *}\right)$ which satisfy

$e^{c *}=\frac{\rho b \bar{t}+2 \sqrt{m\left(\left(\rho b^{2}+4 k m\right) B-\rho k \bar{t}^{2}\right)}}{\rho b^{2}+4 k m}$,

$a-b e^{c *}-m+r^{c *} \ln \frac{\exp \left(2 m / r^{c *}\right)-1}{2 m / r^{c *}}=\tau$.

The optimal utility of the centralized system is $\Pi^{c *}=0$.

Proof: See appendix $C$.

The above proposition shows the optimal level of effort and the minimized tardiness risk. It can be found that the logistics system only obtains reservation utility to minimize the tardiness risk.

\section{Decentralized system under 3PL's cost information symmetric}

In this section, we assume that the 3PL's cost coefficient $k$ is fully observable to the 4PL, while the effort level of 3PL is unobservable to the 4PL, so we have a moral hazard problem.

\subsection{Both the 3PL and 4PL are risk neutral (Case A)}

\subsubsection{Optimal response of $3 P L$}

Given any pair of $(\alpha, \beta)$, the 3PL's profit is function can be expressed as follows:

$$
\pi^{t}(e)=\alpha-\beta \rho[\tilde{t}-\tau]^{+}-k e^{2} .
$$

The expected utility of $3 P L$ is

$$
\Pi^{t}(e)=\alpha-\beta \rho E\left([\tilde{t}-\tau]^{+}\right)-k e^{2} .
$$

After replacing $E\left([\tilde{t}-\tau]^{+}\right)$, Eq. (11) can be finally derived as

$$
\Pi^{t}(e)=\alpha-\frac{\rho(a-b e+m-\tau)^{2} \beta}{4 m}-k e^{2} .
$$

The objective of the 3PL is to maximizes expected utility $\Pi^{t}$. Let $e^{S A *}$ represents the optimal effort level of the 3PL and can be obtained by solve $e^{S A *}=\arg \max \Pi^{t}(e)$.

Lemma 2. Given the contract terms $(\alpha, \beta)$, the 3PL's effort level is $e^{S A *}=\frac{b \rho \beta(a+m-\tau)}{b^{2} \rho \beta+4 k m}$. The $3 P L$ 's effort decision e increases in the penalty coefficient $\beta$.

Proof: See appendix D.

From Lemma 2, we can find that when the penalty coefficient is zero, the 3PL has no desire to exert any other effort. 


\subsubsection{Optimal response of $4 P L$}

The objective of the 4PL is to minimizes the tardiness risk. The total payment given by the 4PL to the 3PL is $\alpha-\beta \rho[\tilde{t}-\tau]^{+}$, where $\beta \rho[\tilde{t}-\tau]^{+}$is the penalty the 4PL imposed on 3PL. By given the triple $\left(\alpha, \beta, e^{S A *}\right)$, the 4PL's profit function can be expressed as follows:

$$
\pi^{f}\left(\alpha, \beta, e^{S A *}\right)=B-\left(\alpha-\beta \rho[\tilde{t}-\tau]^{+}\right)-\rho[\tilde{t}-\tau]^{+} .
$$

The expected utility of $4 \mathrm{PL}$ is

$$
\Pi^{f}\left(\alpha, \beta, e^{S A *}\right)=B-\alpha-\frac{\rho(1-\beta)(a-b e+m-\tau)^{2}}{4 m}
$$

The objective of the 4PL is to minimize the tardiness risk and the contracting problem with the $3 \mathrm{PL}$ can be formulated as follows:

$$
\begin{aligned}
& \min _{\alpha, \beta} r, \\
& \text { subject to } \\
& C_{r}(\tilde{t}) \leq \tau \\
& \Pi^{f}\left(\alpha, \beta, e^{S A *}\right) \geq 0 \\
& \Pi^{t}\left(\alpha, \beta, e^{S A *}\right) \geq 0 \\
& \alpha, \beta, e^{S A *} \geq 0, r>0 .
\end{aligned}
$$

Constraint (16) represents the certainty equivalent of delivery time which should be less than the delivery lead time $\tau$. Constraints (17) and (18) ensure that the 4PL and 3PL earn at least their reservation utilities so that they are willing to cooperate. The above minimization problem can be solved by using Karush-Kuhn-Tucker condition. The optimal solution to the above problem is as follows.

Proposition 2 If $\frac{\rho k \bar{t}^{2}}{\rho b^{2}+4 k m} \leq B \leq \frac{\left(4 k m(a-m)-\rho b^{2}(2 m-\tau)\right)^{2}}{4 b^{2} m\left(\rho b^{2}+4 k m\right)}+\frac{\rho k \bar{t}^{2}}{\rho b^{2}+4 k m}$, the optimal penalty coefficient is

$\beta^{s *}=\frac{2 k\left(2 m b B+\bar{t} \sqrt{m\left(4 k m B-\rho\left(k \bar{t}^{2}-b^{2} B\right)\right)}\right)}{b \rho\left(k \bar{t}^{2}-b^{2} B\right)} ;$

The optimal fixed payment is

$\alpha^{S *}=\frac{k \bar{t}\left(2 m b B+\bar{t} \sqrt{\left.m\left(4 k m B-\rho\left(k \bar{t}^{2}-b^{2} B\right)\right)\right)}\right.}{b\left(2 k m \bar{t}+b \sqrt{m\left(4 k m B-\rho\left(k \bar{t}^{2}-b^{2} B\right)\right)}\right)} ;$

The 3PL's optimal effort level is

$e^{s *}=\frac{2 m b B+\bar{t} \sqrt{m\left(4 k m B-\rho\left(k \bar{t}^{2}-b^{2} B\right)\right)}}{2 k m \bar{t}+b \sqrt{m\left(4 k m B-\rho\left(k \bar{t}^{2}-b^{2} B\right)\right)}} ;$

The optimal $r^{s *}$, which cannot be derived as closed-form expression, satisfies the following equation:

$r^{S *} \ln \frac{\exp \left(2 m / r^{S^{*}}\right)-1}{2 m / r^{S *}}=2 m-\frac{4 k m \bar{t}}{b^{2} \rho \beta^{S *}+4 k m} ;$

The 4PL's optimal expected utility is $\Pi^{f s *}=0$, the $3 P L$ 's optimal expected utility is $\Pi^{t s *}=0$.

Proof: See appendix E.

From proposition 2, we can find that the 4PL and 3PL only obtain their reservation utilities, as all their other utilities are used to reduce the tardiness risk.

Lemma 3. The optimal tardiness risk $r^{{ }^{*}}$ decreases in the optimal penalty coefficient $\beta^{s *}$, and is independent in the optimal fix payment $\alpha^{s *}$.

Proof: See appendix F.

Substituting the expression of $\alpha^{S^{*}}$ into the risk equation, we have $r^{S *} \ln \frac{\exp \left(2 m / r^{S *}\right)-1}{2 m / r^{S *}}=2 m-$ $\frac{2 m\left(k \bar{t}^{2}-b^{2} B\right)}{b \sqrt{m\left(4 k m B-\rho\left(k \bar{t}^{2}-b^{2} B\right)\right)}+2 k m \bar{t}}$, so the following proposition holds: 
Proposition 3 The tardiness risk $r^{s *}$ increases in $\rho, k$ and $a$, and decreases in $B$ and $b$.

Proof: See appendix $G$.

For a certain tardiness risk, a larger unit time penalty $\rho$ means that the $4 \mathrm{PL}$ will compensate the client more. The larger the 3PL's cost coefficient $k$, the more it costs him to complete a certain delivery task, which will lead to a larger tardiness risk. To reduce the risk of tardiness, the 4PL only obtains reservation utility, so the more the client pays the 4PL, the less tardiness risk for the 4PL. The larger the 3PL's effort output coefficient $b$ means that the 3PL saves more time by paying a certain amount of effort. The 3PL with a larger initial delivery time $a$ and smaller effort output coefficient $b$ means that the 3PL is less capable and has a larger tardiness risk.

Comparing results in the symmetric scenario with that in the centralized system, we propose the following proposition.

Proposition 4 The effort levels and tardiness risks satisfy $e^{s *}=e^{c *}, r^{s *}=r^{c *}$.

Proof: See appendix $H$.

From proposition 4, the minimized tardiness risk and the optimal effort level are equal in both cases. Proposition 4 shows that under complete information, the 4PL has the same tardiness risk that she would get if she was carrying out the task herself (with the same cost function as the 3PL), i.e., delegation is costless for the 4PL.

\subsection{The 3PL is risk averse and $4 P L$ is risk neutral (Case B)}

In case $\mathrm{B}$, the 3PL is risk averse and determines the optimal effort level to maximize his mean-variance objective function (Chiu and Choi, 2016), which is shown in Eq. (19).

$$
\widehat{\Pi}^{t}(\alpha, \beta, e)=E\left(\pi^{t}\right)-\delta \operatorname{var}\left(\pi^{t}\right),
$$

where $\delta$ is the degree of the risk aversion. From $\operatorname{var}[a-b e+\varepsilon-\tau]^{+}=\frac{(a-b e-\tau+m)^{3}}{6 m}-\frac{(a-b e-\tau+m)^{4}}{16 m^{2}}$, we have

$$
\begin{aligned}
& \widehat{\Pi}^{t}=\alpha-\frac{\rho(a-b e-\tau+m)^{2} \beta}{4 m}-\frac{\delta \rho^{2} \beta^{2}(a-b e-\tau+m)^{3}}{2 m}\left(\frac{1}{3}-\frac{a-b e-\tau+m}{8 m}\right)-k e^{2} . \\
& \frac{\partial \widehat{\Pi}^{t}}{\partial e}=\frac{b \rho(a-b e-\tau+m) \beta}{2 m}+\frac{\delta b \rho^{2} \beta^{2}(a-b e-\tau+m)^{2}}{2 m}\left(1-\frac{a-b e-\tau+m}{2 m}\right)-2 k e
\end{aligned}
$$

Let $\frac{\partial \widehat{\Pi}^{t}}{\partial \mathrm{e}}=0$, we found that we cannot obtain the closed-form expression of $e$. Even though we cannot derive the closed-form expression of $e, \frac{\partial \widehat{\Pi}^{t}}{\partial \mathrm{e}}=0$ be solved by using numerical optimization methods. To determine the contract terms $(\alpha, \beta)$ and to minimize the tardiness risk, the 4PL's contracting problem with the risk-averse 3PL can be formulated as

$$
\begin{aligned}
& \underset{\alpha, \beta}{\min r}, \\
& \text { subject to } \\
& C_{r}(\tilde{t}) \leq \tau, \\
& \Pi^{f}(\alpha, \beta, e) \geq 0, \\
& \widehat{\Pi}^{t}(\alpha, \beta, e) \geq 0, \\
& e^{*}=\underset{e}{\arg \max } \widehat{\Pi}^{t}(\alpha, \beta, e), \\
& \alpha, \beta, e \geq 0, r>0 .
\end{aligned}
$$

Constraint (23) ensures that the certainty equivalent of delivery time should be less than the delivery lead time $\tau$. Constraints (24) and (25) ensure that the 4PL and 3PL earn at least their reservation utilities, so that they are willing to cooperate with each other. Constraint (26) represents that the 3PL determines his optimal effort to obtain maximum expected profit. We adopt the numerical analysis method to investigate the results. Considering the range of parameters provided by Huang et al. (2019), we set the model parameters as follows: $a=8, b=2, m=6, \tau=7, \rho=6, B=20$, and $k=\{7,7.5,8\}$. In the following parts, four experiments are presented. In the first experiment, the penalty coefficient is investigated. The experimental result is shown in Fig. 2. In Fig. 2, the penalty coefficient decreases in the degree of risk aversion for $k$ equal to 7, 7.5, and 8, respectively. In addition, in Fig. 2, the penalty coefficient decreases in the cost coefficient. Because when the cost coefficient is larger, the 4PL should reduce the penalty to the 3PL to ensure that he obtains the reservation utility. Thus, for a low-type $3 \mathrm{PL}$ with a higher cost coefficient, the 4PL imposes a higher penalty coefficient. 


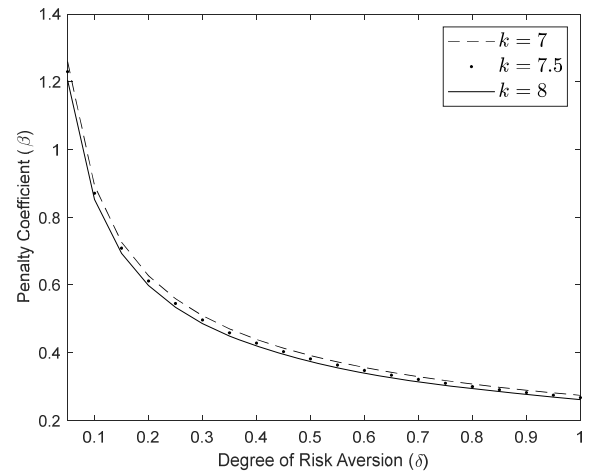

Fig. 2. The effect of degree of risk aversion on the penalty coefficient

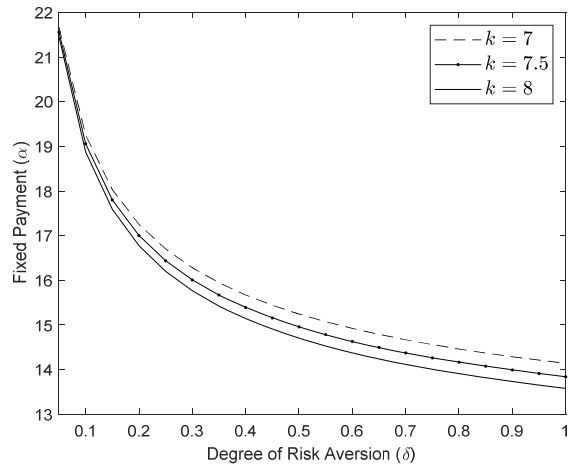

Fig. 3. The effect of degree of risk aversion on the fixed payment

The second experiment is presented in Fig. 3 for exploring the fixed payment affected by the degree of risk aversion and cost coefficient. Fig. 3 indicates that the fixed payment decreases in the degree of risk aversion. Because with the degree of risk aversion increases, the penalty coefficient and effort level decrease, then the 4PL will reduce the fixed payment, ensuring that the 3PL still only achieves the reservation utility. The fixed payment increases in the cost coefficient. The third experiment is presented in Fig. 4 for exploring the 3PL's effort level. Fig. 4 indicates that the effort level decreases in the degree of risk aversion. The effort level decreases in the cost coefficient. Because when the cost coefficient is larger, the 3PL can only make less effort to ensure that he obtains the reservation utility.

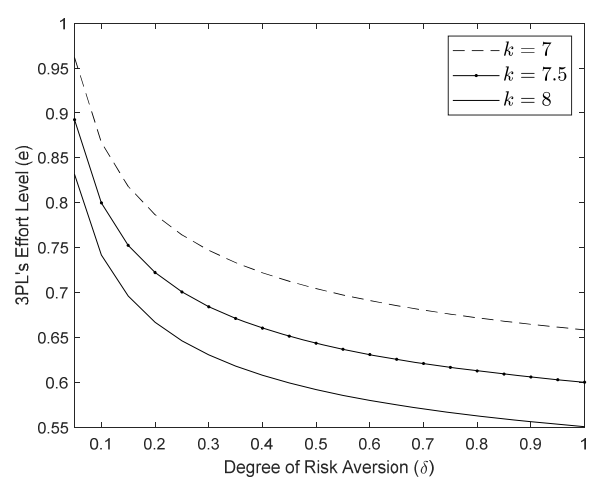

Fig. 4. The effect of degree of risk aversion on the effort level

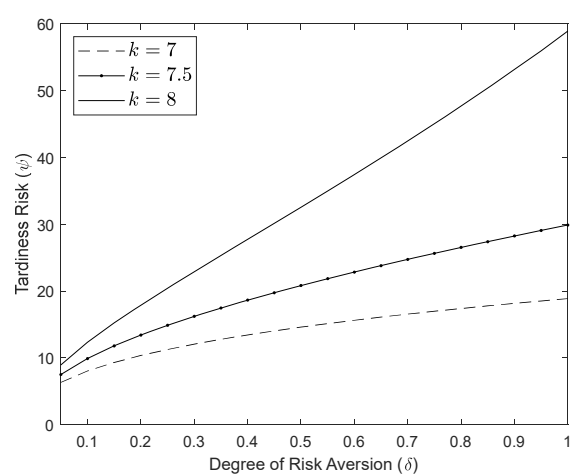

Fig. 5. The effect of degree of risk aversion on tardiness risk

The last experiment is presented in Fig. 5 for exploring the tardiness risk affected by risk aversion and cost coefficient. In Fig. 5 , we find that the tardiness risk is increasing in the degree of risk aversion, which shows that 3PL's risk aversion leads the 4PL to weaken incentives to the 3PL and have to take more tardiness risk. The tardiness risk is increasing in the cost coefficient. Because with a smaller cost coefficient, the 3PL can make more effort and the tardiness risk is smaller. As the cost coefficient increases, the difference between tardiness risks corresponding to the different cost coefficient also increases.

\section{Decentralized system under asymmetric information}

The cost of 3PL is related to many items, such as warehousing, distribution operatives, transportation, and packaging, then the cost coefficient of the 3PL is unobservable to the 4PL. Thus, this section assumes that the 3PL's cost coefficient $k$ is asymmetric information. We assume that the 3PL can be the high type with a low-cost coefficient $k_{h}$ or the low type with a high-cost coefficient $k_{l}$, with $k_{h}<k_{l}$. The 4PL is the principal who only knows the probability $v$, but not the actual type. Also, the effort level of 3PL is unobservable to the 4PL, then the problem considered in this section is a mixed moral hazard and adverse selection problem.

\subsection{Both the 3PL and 4PL are risk neutral (Case A)}

In case A, both the 3PL and 4PL are risk neutral. Based on the two types of 3PL, the penalty and the fixed payment can be represented as $\left(\alpha_{h}, \beta_{h}\right)$ and $\left(\alpha_{l}, \beta_{l}\right)$. Considering that the 4PL determines the menu of contracts $\left\{\left(\alpha_{h}, \beta_{h}\right) ;\left(\alpha_{l}, \beta_{l}\right)\right\}$ to minimize the tardiness risk. The problem can be formulated as 


$$
\begin{aligned}
& \min _{\left(\alpha_{h}, \beta_{h}\right),\left(\alpha_{l}, \beta_{l}\right)} v r_{h}+(1-v) r_{l}, \\
& \text { subject to } \\
& C_{r_{h}}(\tilde{t}) \leq \tau, \\
& C_{r_{l}}(\tilde{t}) \leq \tau, \\
& \Pi^{f}\left(\alpha_{h}, \beta_{h}, e_{h} \mid k_{h}\right) \geq 0, \\
& \Pi^{f}\left(\alpha_{l}, \beta_{l}, e_{l} \mid k_{l}\right) \geq 0, \\
& \Pi^{t}\left(\alpha_{h}, \beta_{h}, e_{h} \mid k_{h}\right) \geq 0, \\
& \Pi^{t}\left(\alpha_{l}, \beta_{l}, e_{l} \mid k_{l}\right) \geq 0, \\
& e_{h}^{*}=\underset{e_{h}}{\operatorname{argax} \Pi^{t}\left(\alpha_{h}, \beta_{h}, e_{h} \mid k_{h}\right),} \\
& e_{l}^{*}=\underset{e_{l}}{\operatorname{argax}} \Pi^{t}\left(\alpha_{l}, \beta_{l}, e_{l} \mid k_{l}\right), \\
& \Pi^{t}\left(\alpha_{h}, \beta_{h}, e_{h} \mid k_{h}\right) \geq \Pi^{t}\left(\alpha_{l}, \beta_{l}, e_{l} \mid k_{h}\right), \\
& \Pi^{t}\left(\alpha_{l}, \beta_{l}, e_{l} \mid k_{l}\right) \geq \Pi^{t}\left(\alpha_{h}, \beta_{h}, e_{h} \mid k_{l}\right), \\
& \alpha_{h}, \beta_{h}, e_{h}, \alpha_{l}, \beta_{l}, e_{l} \geq 0, r_{h}, r_{l}>0 .
\end{aligned}
$$

The objective function (27) minimizes the tardiness risk. Constraints (28-29) represent that the certainty equivalent of $\tilde{t}$ should be less than the delivery lead time $\tau$. Constraints (30-33) ensure that the 4PL and two types of 3PL earn at least their reservation utilities. Constraints (34-35) denote that the two types of 3PL determine their optimal efforts for obtaining the maximum expected utilities. Incentive compatibility constraints (36-37) denote that it's in the best interest of the two types of 3PL to truthfully report their cost coefficients.

The effort level of the 3PL is $e=\frac{b \rho \beta(a+m-\tau)}{b^{2} \rho \beta+4 k m}$, then the utility of 3PL is given by $\Pi^{t}(\alpha, \beta)=\alpha-\frac{k \rho(a+m-\tau)^{2} \beta}{b^{2} \rho \beta+4 k m}$. Let $(\alpha, \beta)$ be a pair of value that satisfies the 3PL utility curve $\Pi^{t}(\alpha, \beta)$, then the $\frac{d \alpha}{d \beta}=\frac{4 k^{2} m \rho(a+m-\tau)^{2}}{\left(b^{2} \rho \beta+4 k m\right)^{2}}>0$ always holds. Define $R=B-$ $\Delta \Pi^{\mathrm{t}}$, where $\Delta \Pi^{\mathrm{t}}=\frac{b^{2} \rho^{2}(a+m-\tau)^{2}\left(k_{l}-k_{h}\right) \beta_{l}^{a * 2}}{\left(b^{2} \rho \beta_{l}^{a *}+4 k_{l} m\right)^{2}}>0$, then we have $B>R$. The optimal solution to the above problem is as follows.

Proposition 5. If $\max \left\{\frac{\rho k_{l} \bar{t}^{2}}{4 k_{l} m+\rho b^{2}}, \frac{\rho k_{h} \bar{t}^{2}}{4 k_{h} m+\rho b^{2}}+\Delta \Pi^{\mathrm{t}}\right\} \leq B \leq \min \left\{\frac{\left(4 k_{l} m(a-m)-\rho b^{2}(2 m-\tau)\right)^{2}}{4 b^{2} m\left(\rho b^{2}+4 k_{l} m\right)}+\right.$ $\left.\frac{\rho k_{l} \bar{t}^{2}}{\rho b^{2}+4 k_{l} m}, \frac{\left(4 k_{h} m(a-m)-\rho b^{2}(2 m-\tau)\right)^{2}}{4 b^{2} m\left(\rho b^{2}+4 k_{h} m\right)}+\frac{\rho k_{h} \bar{t}^{2}}{\rho b^{2}+4 k_{h} m}+\Delta \Pi^{\mathrm{t}}\right\}$, the optimal penalty coefficients are

$\beta_{h}^{a *}=\frac{2 k_{h}\left(2 m b R+\bar{t} \sqrt{m\left(4 k_{h} m R-\rho\left(k_{h} \bar{t}^{2}-b^{2} R\right)\right)}\right)}{b \rho\left(k_{h} \bar{t}^{2}-b^{2} R\right)}$,

$\beta_{l}^{a *}=\frac{2 k_{l}\left(2 m b B+\bar{t} \sqrt{\left.m\left(4 k_{l} m B-\rho\left(k_{l} \bar{t}^{2}-b^{2} B\right)\right)\right)}\right.}{b \rho\left(k_{l} \bar{t}^{2}-b^{2} B\right)} ;$

The optimal fixed payments are

$\alpha_{h}^{a *}=\frac{k_{h} \bar{t}\left(2 m b R+\bar{t} \sqrt{m\left(4 k_{h} m R-\rho\left(k_{h} \bar{t}^{2}-b^{2} R\right)\right)}\right)}{b\left(2 k_{h} m \bar{t}+b \sqrt{m\left(4 k_{h} m R-\rho\left(k_{h} \bar{t}^{2}-b^{2} R\right)\right)}\right)}+\Delta \Pi^{\mathrm{t}}$,

$\alpha_{l}^{a *}=\frac{k_{l} \bar{t}\left(2 m b B+\bar{t} \sqrt{\left.m\left(4 k_{l} m B-\rho\left(k_{l} \bar{t}^{2}-b^{2} B\right)\right)\right)}\right.}{b\left(2 k_{l} m \bar{t}+b \sqrt{m\left(4 k_{l} m B-\rho\left(k_{l} \bar{t}^{2}-b^{2} B\right)\right)}\right)} ;$

The minimum risk is

$\psi^{a *}=v r_{h}^{a *}+(1-v) r_{l}^{a *}$,

where

$r_{i}^{a *} \ln \frac{\exp \left(2 m / r_{i}^{a *}\right)-1}{2 m / r_{i}^{a *}}=2 m-\frac{4 k_{i} m \bar{t}}{b^{2} \rho \beta_{i}^{a *}+4 k_{i} m}, i=h, l$

The 3PL's optimal effort level are

$\begin{aligned} e_{h}^{a *} & =\frac{2 m b R+\bar{t} \sqrt{m\left(4 k_{h} m R-\rho\left(k_{h} \bar{t}^{2}-b^{2} R\right)\right)}}{\left.2 k_{h} m \bar{t}+b \sqrt{m\left(4 k_{h} m R-\rho\left(k_{h} \bar{t}^{2}-b^{2} R\right)\right.}\right)}, \\ e_{l}^{a *} & =\frac{2 m b B+\bar{t} \sqrt{m\left(4 k_{l} m B-\rho\left(k_{l} \bar{t}^{2}-b^{2} B\right)\right)}}{2 k_{l} m \bar{t}+b \sqrt{m\left(4 k_{l} m B-\rho\left(k_{l} \bar{t}^{2}-b^{2} B\right)\right)}} .\end{aligned}$

The 4PL's optimal utility is $\Pi^{\text {fa* }}=0$, the low type $3 P L$ with high cost coefficient only obtains reservation utility $\Pi_{l}^{\text {ta* }}=0$, the optimal utility of the high type $3 P L$ with low cost coefficient is $\Pi_{h}^{\text {ta* }}=\Delta \Pi^{\mathrm{t}}$, where $\Delta \Pi^{\mathrm{t}}$ is the information rent.

Proof: See appendix I. 
The high type 3PL under asymmetric information earns information rent which reflects the informational advantage of high type 3PL over low type 3PL. The information rent depends only on the low type 3PL's penalty coefficient given by 4PL, and the information rent increases in the penalty coefficient for the low type 3PL. Comparing the optimal effort level and minimized tardiness risk in the asymmetric scenario with that in the centralized system, we have proposition 6.

Proposition 6 From $B>R$, the effort levels and tardiness risks satisfy $e_{l}^{c *}=e_{l}^{a *}, e_{h}^{c *}>e_{h}^{a *}$, and $r_{l}^{c *}=v r_{h}^{a *}+(1-v) r_{l}^{a *}>$ $r_{h}^{c *}$.

Proof: See appendix J.

From proposition 6, the high type 3PL makes less effort under asymmetric information than in the centralized system, which results in larger tardiness risk $r_{h}^{a *}$. Then, the expected tardiness risk in the decentralized system under asymmetric information is larger than the tardiness risk with a high type 3PL in the centralized system, while is equal to the tardiness risk with a low type 3PL in the centralized system. The increase in the tardiness risk indicates that asymmetric information leads to a reduction in the efficiency of the logistics system. Comparing the optimal penalty coefficients and fixed payments in the decentralized system under asymmetric information with that under symmetric information, we have proposition 7.

Proposition 7. From $B>R$, the penalty coefficients and fixed payments for the low and high type 3PL satisfy

(i) $\beta_{l}^{a *}=\beta_{l}^{S *}, \beta_{h}^{a *}<\beta_{h}^{S *}$,

(ii) $\alpha_{l}^{a *}=\alpha_{l}^{S *}$,

(iii) $\alpha_{h}^{a *}<\alpha_{h}^{S *}$, if $\beta_{h}^{a *}<\frac{4 k m}{b^{2} \rho}+2 ; \alpha_{h}^{a *}>\alpha_{h}^{S *}$, if $\beta_{h}^{a *}>\frac{4 k m}{b^{2} \rho}+2$.

Proof: See appendix $K$.

From proposition 7, for the low type 3PL, the penalty coefficient and fixed payment in the asymmetric scenario are equal to that in the symmetric scenario. For the high type 3PL, the penalty coefficient in the asymmetric scenario is lower than that in the symmetric scenario, while the fixed payment in the symmetric scenario may be smaller or larger than that in the asymmetric scenario. Thus, the penalty coefficient and fixed payment of the high type 3PL are distorted in the asymmetric scenario.

\subsection{The 3PL is risk averse and 4PL is risk neutral (Case B)}

In this case, both types choose the optimal effort levels to maximize their mean-variance objective function (Chiu \& Choi, 2016).

$\widehat{\Pi}^{t}(\alpha, \beta, e \mid k)=E\left(\pi^{t}\right)-\delta \operatorname{var}\left(\pi^{t}\right)$,

where $\delta$ is his degree of the risk aversion. From $\operatorname{var}[a-b e+\varepsilon-\tau]^{+}=\frac{(a-b e-\tau+m)^{3}}{6 m}-\frac{(a-b e-\tau+m)^{4}}{16 m^{2}}$, we have

$$
\widehat{\Pi}^{t}=\alpha-\frac{\rho(a-b e-\tau+m)^{2} \beta}{4 m}-\frac{\delta \rho^{2} \beta^{2}(a-b e-\tau+m)^{3}}{2 m}\left(\frac{1}{3}-\frac{a-b e-\tau+m}{8 m}\right)-k e^{2} \text {. }
$$

To minimize the tardiness risk, the 4PL's contracting problem with the risk-averse 3PL can be formulated as

$$
\begin{aligned}
& \min _{\left(\alpha_{h}, \beta_{h}\right),\left(\alpha_{l}, \beta_{l}\right)} v r_{h}+(1-v) r_{l}, \\
& \text { subject to } \\
& C_{r_{h}}(\tilde{t}) \leq \tau, \\
& C_{r_{l}}(\tilde{t}) \leq \tau \text {, } \\
& \Pi^{f}\left(\alpha_{h}, \beta_{h}, e_{h} \mid k_{h}\right) \geq 0 \text {, } \\
& \Pi^{f}\left(\alpha_{l}, \beta_{l}, e_{l} \mid k_{l}\right) \geq 0 \text {, } \\
& \widehat{\Pi}^{t}\left(\alpha_{h}, \beta_{h}, e_{h} \mid k_{h}\right) \geq 0 \text {, } \\
& \widehat{\Pi}^{t}\left(\alpha_{l}, \beta_{l}, e_{l} \mid k_{l}\right) \geq 0 \text {, } \\
& e_{h}^{*}=\underset{e_{h}}{\arg \max } \widehat{\Pi}^{t}\left(\alpha_{h}, \beta_{h}, e_{h} \mid k_{h}\right) \text {, } \\
& e_{l}^{*}=\underset{e_{l}}{\arg \max } \widehat{\Pi}^{t}\left(\alpha_{l}, \beta_{l}, e_{l} \mid k_{l}\right) \text {, } \\
& \widehat{\Pi}^{t}\left(\alpha_{h}, \beta_{h}, e_{h} \mid k_{h}\right) \geq \widehat{\Pi}^{t}\left(\alpha_{l}, \beta_{l}, e_{l} \mid k_{h}\right) \text {, } \\
& \widehat{\Pi}^{t}\left(\alpha_{l}, \beta_{l}, e_{l} \mid k_{l}\right) \geq \widehat{\Pi}^{t}\left(\alpha_{h}, \beta_{h}, e_{h} \mid k_{l}\right) \text {, } \\
& \alpha_{h}, \beta_{h}, e_{h}, \alpha_{l}, \beta_{l}, e_{l} \geq 0, r_{h}, r_{l}>0 \text {. }
\end{aligned}
$$


The objective function (40) is to minimize the expected tardiness risk. Constraints (41-42) represent that the certainty equivalent of $\tilde{t}$ should be less than the delivery lead time $\tau$. Participation constraints (43-46) ensure that the 4PL and two types of 3PL earn at least their reservation utilities. Constraints (47-48) represent that the and two types of 3PL determine their optimal efforts to obtain maximum expected utilities. Incentive compatibility constraints (49-50) denote that it's in the best interest of the and two types of 3PL to truthfully report their cost coefficients. Due to it is impossible to derive the closedform expression of tardiness risk, optimal effort level, fixed payment and penalty coefficient when the 3PL is risk averse. Considering the parameter setting of Huang et al. (2019), we set the model parameters as following: $a=8, b=2, m=6, \tau=$ $7, \rho=6, v=0.5, B=20, k_{h}=6$ and $k_{l}=\{7,7.5,8\}$. Fig. 6 indicates that the tardiness risk increases in the degree of risk aversion under asymmetric information. When the degree of risk aversion is given, the larger the cost coefficient, the larger the tardiness risk. Comparing Fig. 5 with Fig. 6, when the degree of risk aversion is given, the tardiness risk under symmetric information in Fig. 5 is greater than that under asymmetric information in Fig. 6.

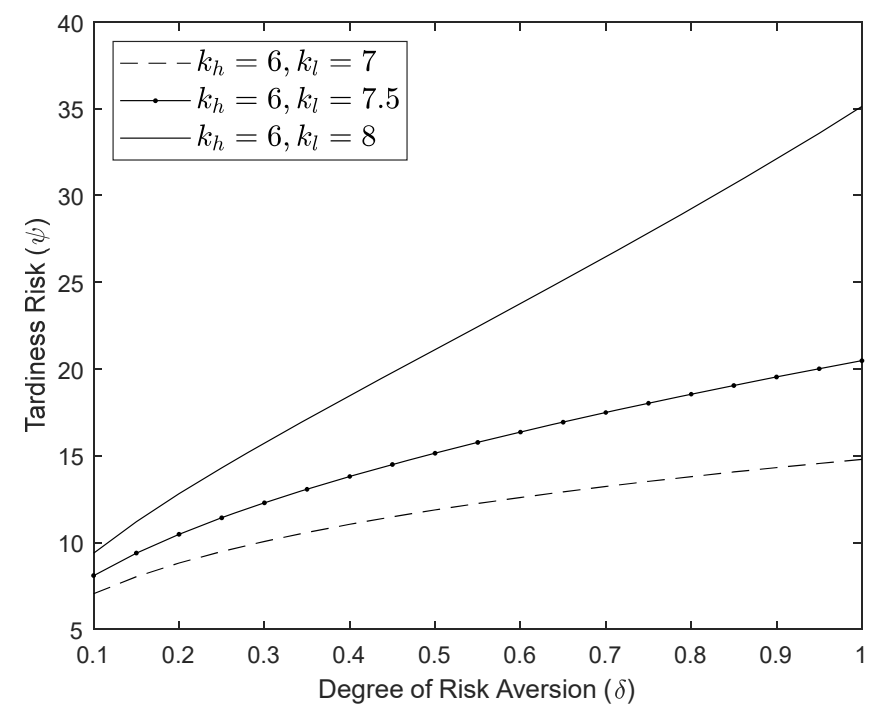

Fig. 6. The effect of degree of risk aversion on tardiness risk under asymmetric information

\section{Numerical results}

In this section, we investigate the 3PL's optimal effort level and the tardiness risk in the centralized system and asymmetric scenario, and the optimal penalty coefficient and fixed payment in the symmetric and asymmetric scenario. We set the model parameters as follows: $a=8, b=2, m=1.5, \tau=6, B=12, k_{l}=5, k_{h}=4, v=0.5$, and $\rho$ varies from 5.4 to 9 . Fig. 7 shows that the penalty coefficient for the low type 3PL in the symmetric scenario is the same as that in the asymmetric scenario, while the penalty coefficient for the high type 3PL in the asymmetric scenario lower than that in the symmetric scenario. Fig. 8 shows that the fixed payment given to the low type 3PL in the symmetric scenario is the same as that in the asymmetric scenario, while the fixed payment given to the high type 3PL in the asymmetric scenario larger than that in the symmetric scenario.

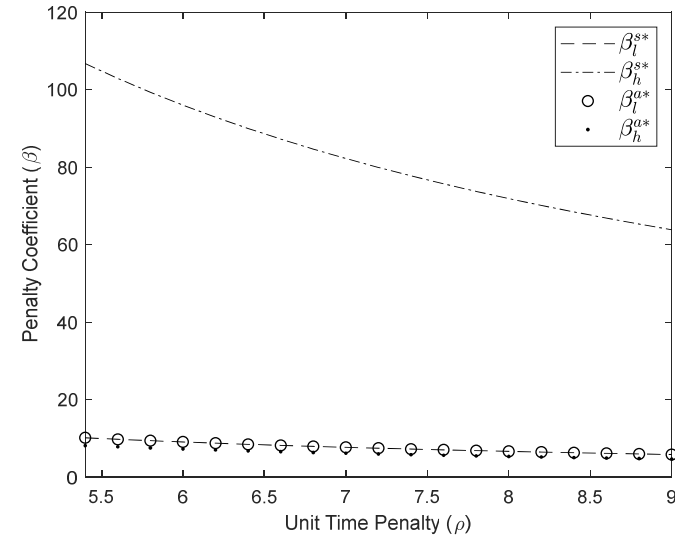

Fig. 7. The penalty coefficient in the symmetric and asymmetric scenario

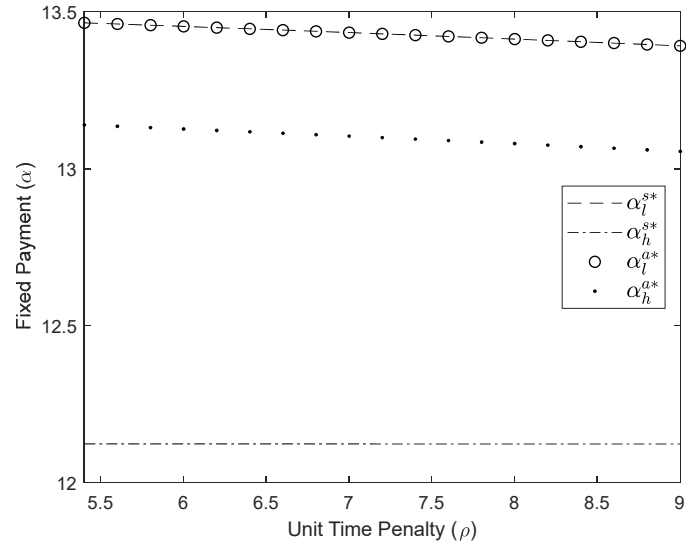

Fig. 8. The fixed payment in the symmetric and asymmetric scenario 
Fig. 9 reveals that the low-type 3PL in a centralized system makes the same effort like that in the asymmetric scenario, while the high-type 3PL in a centralized system makes more effort than that in the asymmetric scenario. Therefore, the effort level of the high type 3PL is distorted under asymmetric scenarios. Fig. 10 shows that, for a low type 3PL, the tardiness risk in the asymmetric scenario is equal to that in the centralized system. For a high type 3PL, the tardiness risk in the asymmetric scenario is larger than that in the centralized system.

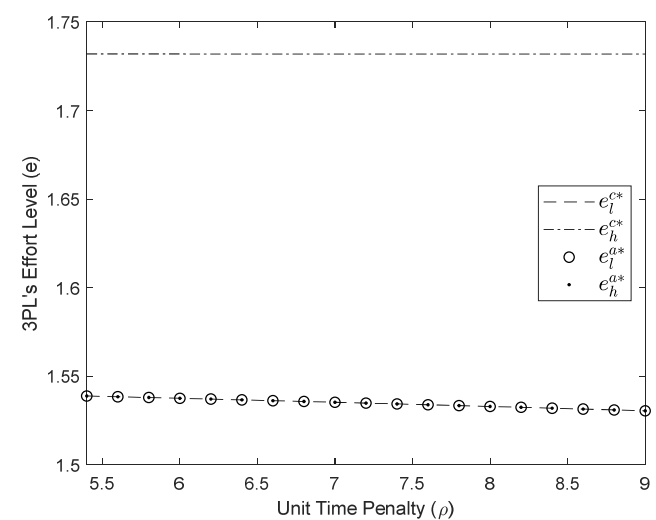

Fig. 9. The 3PL's effort level in centralized system and asymmetric scenario

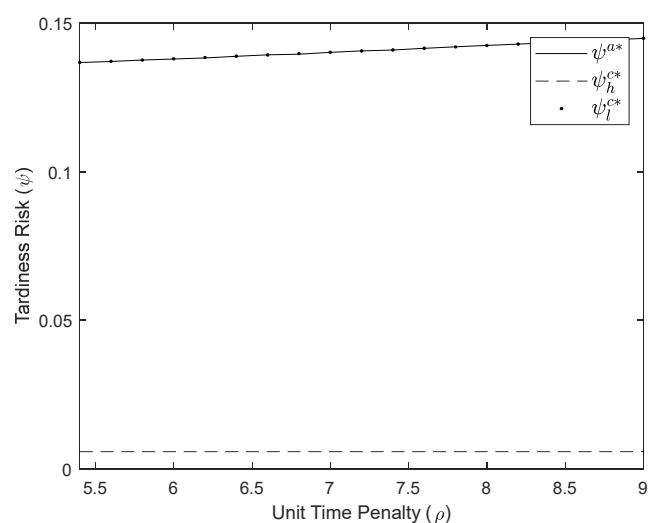

Fig. 10. The tardiness risk in centralized system and asymmetric scenario

\section{Conclusion}

In this paper, we investigate the optimal contracting problem for a fourth party logistics company (4PL) in the presence of the third logistics company (3PL) tardiness risk. To measure the magnitude of tardiness risk and the probability of tardiness risk occurrence, we adopt a tardiness risk index. With the index the 4PL designs incentive contracts that minimize the tardiness risk by motivating the 3PL to make more efforts in the delivery. Considering the 3PL's unobserved cost coefficient information and risk-averse attitude, we propose contracts in the symmetric and asymmetric scenario when 3PL is risk-neutral and risk-averse, respectively.

First, we study the risk management model for a centralized system, which gives a benchmark for the effort level and tardiness risk. Second, when the 3PL is risk neutral, the 3PL's effort level and tardiness risk in the symmetric scenario are equal to that in the centralized system, then the minimized tardiness risk guarantees the reduction of maximum probability of tardiness risk occurrence. Under asymmetric information, the effort level of the high-type 3PL in the decentralized system is smaller than that in the centralized system, and the effort level of the low-type 3PL in the decentralized system is equal to that in the centralized system. Thus, the asymmetry of cost coefficient information gives the high type 3PL an information rent, and leads to an increase of the tardiness risk. Finally, when the 3PL is risk averse, numerical experiments show that the tardiness risk increases, while the effort level, penalty coefficient and fixed payment decrease in the degree of risk aversion. Therefore, the risk aversion of 3PL makes the 4PL take more tardiness risk.

This paper can provide some management implications for the 4PL, such as Cainiao, especially when the 3PL cannot meet the delivery lead time required by the client. To reduce the 3PL's tardiness risk and the maximum probability of the risk occurrence, the 4PL platform can design the fixed payment and penalty contract that takes into account the tardiness risk index. For the 4PL platform, the proposed contract design scheme benefits the mitigation of the tardiness risk and reduces compensation to the client for delivery time.

Several directions can be extended for future studies, which are summarized as follows: (1) the contract under asymmetric information cannot coordinate the system. Other types of contracts under symmetric information can be explored; (2) this paper only considers one 3PL. There may be more than one 3PL on the market for the client to choose from, and it would be fascinating to study the competition among 3PL companies when designing the contract.

\section{Acknowledgments}

We would like to express our great thanks to the anonymous reviewers and the editor-in-chief for providing us valuable comments and suggestions. This work is supported by the NSFC Major International (Regional) Joint Research Project Grant No. 71620107003; the Liaoning Revitalizing Talent Program No. XLYC1802115; the Fundamental Research Funds for State Key Laboratory of Synthetical Automation for Process Industries Grant No. 2013ZCX11; the 111 Incubating Program of Overseas Expert Introduction (BC2018010); the "High-level Overseas Expert" Introduction Program (G20190006026). This work was also supported in part by the National Natural Science Foundation of China under Grant Nos. 62173076, 71671032 
and 71801191, the National Key Research and Development Program of China under Grant No. 2020YFB1708200, and the Fundamental Research Funds for the Central Universities under Grant No. N180408019.

\section{References}

Adulyasak, Y., \& Jaillet, P. (2016). Models and algorithms for stochastic and robust vehicle routing with deadlines. Transportation Science, 50(2), 608-626.

Alp, O., Erkip, N. K., \& Güllü, R. (2003). Outsourcing logistics: Designing transportation contracts between a manufacturer and a transporter. Transportation science, 37(1), 23-39.

Aumann, R. J., \& Serrano, R. (2008). An economic index of riskiness. Journal of Political Economy, 116(5), 810-836.

Bade, D. J., \& Mueller, J. K. (1999). New for the millennium: 4PL trademark. Transportation and Distribution 40(2): 78-81.

Banciu, M., \& Mirchandani, P. (2013). New results concerning probability distributions with increasing generalized failure rates. Operations Research, 61(4), 925-931.

Bureau, P.S.P., 2019. Prc post bureau circular on consumer appeals of the postal service in december 2018. http://www.spb.gov.cn/xw/dtxx 15079/201901/t20190128 1756948.html. (Accessed on 01/12/2021).

Cai, X., Chen, J., Xiao, Y., Xu, X., \& Yu, G. (2013). Fresh-product supply chain management with logistics outsourcing. Omega-International Journal of Management Science, 41(4), 752-765.

Cainiao, 2016. Cainiao network overview. https://www.alizila.com/wp-content/uploads/2016/09/Cainiao-Factsheet.pdf? x95431. (Accessed on 01/12/2021).

Çetinkaya, F., Yeloğlu, P., \& Çatmakaş, H. (2021). Customer order scheduling with job-based processing on a single-machine to minimize the total completion time. International Journal of Industrial Engineering Computations, 12(3), 273-292.

Chen, F. Y., Hum, S. H., \& Sun, J. (2001). Analysis of third-party warehousing contracts with commitments. European Journal of Operational Research, 131(3), 603-610.

Chiu, C. H., \& Choi, T. M. (2016). Supply chain risk analysis with mean-variance models: A technical review. Annals of Operations Research, 240(2), 489-507.

Choi, T. M., Chiu, C. H., \& Chan, H. K. (2016). Risk management of logistics systems. Transportation Research Part E: Logistics and Transportation Review, 90: 1-6.

Gong, F., Kung, D. S., \& Zeng, T. (2018). The impact of different contract structures on IT investment in logistics outsourcing. International Journal of Production Economics, 195, 158-167.

Govindan, K., \& Chaudhuri, A. (2016). Interrelationships of risks faced by third party logistics service providers: A DEMATEL based approach. Transportation Research Part E: Logistics and Transportation Review, 90, 177-195.

Heckmann, I., Comes, T., \& Nickel, S. (2015). A critical review on supply chain risk-Definition, measure and modeling. Omega-International Journal of Management Science, 52, 119-132.

Huang, H., Shen, X., \& Xu, H. (2016). Procurement contracts in the presence of endogenous disruption risk. Decision Sciences, 47(3), 437-472.

Huang, M., Tu, J., Chao, X., \& Jin, D. (2019). Quality risk in logistics outsourcing: A fourth party logistics perspective. European Journal of Operational Research, 276(3), 855-879.

Jaillet, P., Qi, J., \& Sim, M. (2016). Routing optimization under uncertainty. Operations research, 64(1), 186-200.

Laffont, J. J., and Martimort, D. (2002). The Theory of Incentives: The Principal-Agent Model. Princeton: Princeton University Press.

Lim, W. S. (2000). A lemons market? An incentive scheme to induce truth-telling in third party logistics providers. European Journal of Operational Research, 125(3), 519-525.

Liu, W., \& Wang, Y. (2015). Quality control game model in logistics service supply chain based on different combinations of risk attitude. International Journal of Production Economics, 161, 181-191.

Liu, W., Xie, D., Liu, Y., \& Liu, X. (2015). Service capability procurement decision in logistics service supply chain: A research under demand updating and quality guarantee International Journal of Production Research, 53(2), 488-510.

Liu, X., Zhang, K., Chen, B., Zhou, J., \& Miao, L. (2018). Analysis of logistics service supply chain for the One Belt and One Road initiative of China. Transportation Research Part E: Logistics and Transportation Review, 117, 23-39.

Moon, I., Xu, J., Feng, X., \& Ruan, X. (2020). Cooperative sales promotion with a point-sharing policy: Advantages and limitations. Omega-International Journal of Management Science, 94, 102038. (In press)

Ogier, M., Chan, F. T., Chung, S. H., Cung, V. D., \& Boissière, J. (2015). Decentralised capacitated planning with minimalinformation sharing in a 2-echelon supply chain. International Journal of Production Research, 53(16), 4927-4950.

Ogier, M., Cung, V. D., Boissière, J., \& Chung, S. H. (2013). Decentralised planning coordination with quantity discount contract in a divergent supply chain. International Journal of Production Research, 51(9), 2776-2789.

Wang, H., Tan, J., Guo, S., \& Wang, S. (2018). High-value transportation disruption risk management: Shipment insurance with declared value. Transportation Research Part E: Logistics and Transportation Review, 109, 293-310.

Wu, Q., Mu, Y., \& Feng, Y. (2015). Coordinating contracts for fresh product outsourcing logistics channels with power structures. International Journal of Production Economics, 160, 94-105.

Wang, Y., Wallace, S. W., Shen, B., \& Choi, T. M. (2015). Service supply chain management: A review of operational models. European Journal of Operational Research, 247(3), 685-698.

Yamada, T., Nagano, M., \& Miyata, H. (2021). Minimization of total tardiness in no-wait flowshop production systems with preventive maintenance. International Journal of Industrial Engineering Computations, 12(4), 415-426. 
Zhang, M., Fu, Y., Zhao, Z., Pratap, S., \& Huang, G. Q. (2019). Game theoretic analysis of horizontal carrier coordination with revenue sharing in E-commerce logistics. International Journal of Production Research, 57(5), 1524-1551.

Zhang, M., Pratap, S., Huang, G. Q., \& Zhao, Z. (2017). Optimal collaborative transportation service trading in B2B ecommerce logistics. International Journal of Production Research, 55(18), 5485-5501.

Zhou, Y., \& Kim, K. H. (2019). Optimal concession contract between a port authority and container-terminal operators by revenue-sharing schemes with quantity discount. Maritime Policy \& Management, 1-22. (In press)

Zhou, Y., \& Kim, K. H. (2020). Optimal parameters in concession contracts between container terminal operators and investors. International Journal of Logistics Research and Applications, 23(6), 602-625. 
Appendix A: The derivation of $E\left([\tilde{\boldsymbol{t}}-\tau]^{+}\right)$

When $\varepsilon$ follows the uniform distribution $U(-m, m)$, the expression of $E\left([\tilde{t}-\tau]^{+}\right)$is presented as follows: $E\left([\tilde{t}-\tau]^{+}\right)=$ $\int_{-m}^{a-b e-\tau} \frac{1}{2 m}(a-b e-\varepsilon-\tau) d \varepsilon=\left.\frac{1}{2 m}(a-b e-\tau) \varepsilon\right|_{-m} ^{a-b e-\tau}-\left.\frac{1}{4 m} \varepsilon^{2}\right|_{-m} ^{a-b e-\tau}=\frac{1}{2 m}(a-b e-\tau)(a-b e+m-\tau)-$ $\frac{1}{4 m}\left((a-b e-\tau)^{2}-m^{2}\right)=\frac{1}{4 m}(a-b e+m-\tau)^{2} \mathbf{\square}$.

\section{Appendix B: Proof of Lemma 1}

If $r>0$, from the moment generating function, when $\tilde{t}$ follows the uniform distribution $U(a-b e-m, a-b e+m)$, $E(\exp (\tilde{t} / r))=\frac{\exp ((a-b e+m) / r)-\exp ((a-b e-m) / r)}{2 m / r}=\exp ((a-b e-m) / r) \frac{\exp (2 m / r)-1}{2 m / r}$. Then

$$
r \ln E(\exp (\tilde{t} / r))=r((a-b e-m) / r+\ln (\exp (2 m / r)-1)-\ln (2 m / r))
$$

Take the logarithm of both side of the equation (B.1) and then multiply $r$, we can obtain the following equation:

$$
r \ln E(\exp (\tilde{t} / r))=a-b e-m+r(\ln (\exp (2 m / r)-1)-\ln (2 m / r))
$$

After make some basic arithmetic operations of equation (B.2), we can derive that

$r \ln E(\exp (\tilde{t} / r))=a-b e-m+r \ln \frac{\exp (2 m / r)-1}{2 m / r}$

Let $f(r)=r \ln \frac{\exp (2 m / r)-1}{2 m / r}$. In the following, we prove that $f(r)$ is monotone increasing in $r$. The first-order derivative of $f(r)$ with respect to $r$ is given by

$f^{\prime}(r)=\ln (\exp (2 m / r)-1)-\ln (2 m / r)-\frac{\exp (2 m / r)(2 m / r)}{\exp (2 m / r)-1}+1<0$.

Finally, we conclude that when $r<0, f(r)$ is monotone decreasing, and we proof Lemma $1 \mathbf{a}$.

\section{Appendix C: Proof of Proposition 1}

From appendix A, we can obtain that $\mathrm{E}[\tilde{t}-\tau\}]^{+}=\frac{(a-b e+m-\tau)^{2}}{4 m}$. Because the constraint (9) is quadratic with respect to $e$, so constraint (9) is concave in e. Furthermore, let $\mu(e, r)=-(a-b e-m+f(r)-\tau)$, the first-order derivatives of $\mu(e, r)$ with respect to $e$ and $r$ are $\frac{\partial \mu}{\partial e}=b, \frac{\partial \mu}{\partial r}=-\ln (\exp (2 m / r)-1)+\ln (2 m / r)+\frac{\exp (2 m / r)(2 m / r)}{\exp (2 m / r)-1}-1$. Then, the second-order derivatives of $\mu(e, r)$ with respect to $e$ and $r$ are $\frac{\partial^{2} \mu}{\partial e^{2}}=0, \frac{\partial^{2} \mu}{\partial e \partial r}=0, \frac{\partial^{2} \mu}{\partial r^{2}}=\left(4 m^{2} / r^{3}\right) \exp (2 m / r) /(\exp (2 m / r)-1)^{2}+$ $1 / r>0$. The Hessian matrix of $\mu(e, r)$ is semi-positive definite, it follows that $\mu(e, r)$ is concave in $(e, r)$, so the problem is concave in $(e, r)$. Let $\lambda_{1}$ and $\lambda_{2}$ represent the non-negative lagrange multipliers. The Lagrangian function is $L^{c}=r+\lambda_{1}(a-$ $b e-m+f(r)-\tau)+\lambda_{2}\left(R-B+\rho(a-b e+m-\tau)^{2} /(4 m)+k e^{2}\right)$.

$\frac{\partial L^{c}}{\partial e}=\lambda_{1}(-b)+\lambda_{2}(-b \rho(a-b e+m-\tau) /(2 m)+2 k e)=0$,

$\frac{\partial L^{c}}{\partial r}=1+\lambda_{1} f^{\prime}(r)=0$.

Because $f^{\prime}(r)<0$, we have $\lambda_{1}>0$. From $\lambda_{1} \neq 0$, it follows that $\lambda_{2} \neq 0$. Hence, we have $\lambda_{2}=\frac{2 m b \lambda_{1}}{\left(b^{2} \rho+4 k m\right) e-b \rho(a+m-\tau)}>0$. From $\lambda_{1}, \lambda_{2}>0$, we obtain $a-b e-m+f(r)-\tau=0, R-B+\frac{\rho(a-b e+m-\tau)^{2}}{4 m}+k e^{2}=0$. Then we obtain $e^{c *}=$ $\frac{\rho b(a+m-\tau)+2 \sqrt{m\left(\left(\rho b^{2}+4 k m\right) B-\rho k(a+m-\tau)^{2}\right)}}{\rho b^{2}+4 k m}$. To ensure the delivery time is positive, we assume $e^{c *} \leq(a-m) / b$, then we have $B \leq\left(4 k m(a-m)-\rho b^{2}(2 m-\tau)\right)^{2} /\left(4 b^{2} m\left(\rho b^{2}+4 k m\right)\right)+\rho k(a+m-\tau)^{2} /\left(\rho b^{2}+4 k m\right) \quad$ and $\quad 4 k m(a-m) \geq$ $\rho b^{2}(2 m-\tau) \mathbf{\square}$.

\section{Appendix D: Proof of Lemma 2}

From the expression (12), the first derivative of $\Pi^{t}$ with respect to $e$ is given by $\frac{\partial \Pi^{t}(\alpha, \beta, e)}{\partial e}=\frac{b \rho(a-b e+m-\tau) \beta}{2 m}-2 k e$.

Let $\frac{\partial \Pi^{t}(\alpha, \beta, e)}{\partial e}=0$, we can obtain that $\frac{b \rho(a-b e+m-\tau) \beta}{m}-2 k e=0$. After solving this equation $\frac{b \rho(a-b e+m-\tau) \beta}{m}-2 k e=0$, we derive that $e=\frac{b \rho \beta(a+m-\tau)}{b^{2} \rho \beta+4 k m}$ 
Appendix E: Proof of Proposition 2

We substitute $e^{s}$ in expressions (16)-(18) with $\frac{b \rho \beta^{s}(a+m-\tau)}{b^{2} \rho \beta^{s}+4 k m}$. Let $\lambda_{3}, \lambda_{4}$ and $\lambda_{5}$ represent the nonnegative. The Lagrange function is

$$
L^{s}=r+\lambda_{3}\left(a-\frac{b^{2} \rho \beta \bar{t}}{4 k m+b^{2} \rho \beta}-m+f(r)-\tau\right)-\lambda_{5}\left(\alpha-\frac{k \rho \bar{t}^{2} \beta}{4 k m+b^{2} \rho \beta}\right)-\lambda_{4}\left(B-\alpha+\frac{4 k^{2} m \rho(\beta-1) \bar{t}^{2}}{\left(4 k m+b^{2} \rho \beta\right)^{2}}\right) .
$$

Taking the derivative of $L^{s}$ with respect to $r, \alpha$ and $\beta$, we have

$$
\begin{aligned}
& \frac{\partial^{2} L^{S}}{\partial r}=1+\lambda_{3} f^{\prime}(r)=0, \\
& \frac{\partial^{2} L^{S}}{\partial \alpha}=\lambda_{4}-\lambda_{5}=0, \\
& \frac{\partial L^{S}}{\partial \beta}=\lambda_{3}\left(-b^{2} \rho\right)-\lambda_{4} \bar{t} k \rho \frac{4 k m-b^{2} \rho \beta+2 b^{2} \rho}{4 k m+b^{2} \rho \beta}+\lambda_{5} k \rho \bar{t} .
\end{aligned}
$$

The Eq. (E.4) can be simplified by using the condition that $\lambda_{4}=\lambda_{5}$. Then the Eq. (E.4) is reformed as

$$
\frac{\partial L^{S}}{\partial \beta}=-\lambda_{3}+\lambda_{4} \frac{2(a+m-\tau) k \rho(\beta-1)}{4 k m+b^{2} \rho \beta}=0 .
$$

Because $f^{\prime}(r)<0$, we have $\lambda_{3}>0$. From $\lambda_{3} \neq 0$, it follows that $\lambda_{4}=\lambda_{5} \neq 0$. Hence, we have $\lambda_{4}=\lambda_{5}=\frac{\lambda_{3}\left(4 \mathrm{~km}+b^{2} \rho \beta\right)}{2(a+m-\tau) k \rho(\beta-1)}>$ 0 that satisfy $\beta>1$. Then we obtain the desired results. From $4 k m(a-m)>\rho b^{2}(2 m-\tau)$, we have $\frac{\left(4 k m(a-m)-\rho b^{2}(2 m-\tau)\right)^{2}}{4 b^{2} m\left(\rho b^{2}+4 k m\right)}+\frac{\rho k(a+m-\tau)^{2}}{\rho b^{2}+4 k m}-\frac{k(a+m-\tau)^{2}}{b^{2}}=-\frac{\left(4 k m(2 a-\tau)-\rho b^{2}(2 m-\tau)\right)(2 m-\tau)}{4 b^{2} m}<-\frac{(a+m-\tau) \rho(2 m-\tau)^{2}}{4 m(a-m)}<0$ ш.

\section{Appendix F: Proof of Lemma 3}

Let $f(r)=r \ln \frac{\exp (2 m / r)-1}{2 m / r}$ and $g(\beta)=2 m-\frac{4 k m(a+m-\tau)}{b^{2} \rho \beta+4 k m}$. We can easily find that $f(r)=g(\beta)$. Because $a+m-\tau>0$, $g(\beta)$ is monotone increasing in $\beta$. Let $\beta^{\prime}$ and $\beta^{\prime \prime}$ are arbitrary positive values with $\beta^{\prime}>\beta^{\prime \prime}>0, G\left(\beta^{\prime}\right)>G\left(\beta^{\prime \prime}\right)$. $r^{\prime}$ and $r^{\prime \prime}$ corresponds the $\beta^{\prime}$ and $\beta^{\prime \prime}$ with $f\left(\beta^{\prime}\right)=f\left(r^{\prime}\right)$ and $g\left(\beta^{\prime \prime}\right)=f\left(r^{\prime \prime}\right)$. Obvious, $f\left(r^{\prime}\right)>f\left(r^{\prime \prime}\right)$ is hold. Because the function $f(r)$ is monotone increasing in $r$, we can conclude that $r^{\prime}<r^{\prime \prime}$. Finally, we can conclude that when the optimal $\beta$ is decreasing, the optimal $r$ is increasing $\mathbf{m}$

\section{Appendix G: Proof of Proposition 3}

Let $f\left(r^{S^{*}}\right)=r^{s *} \ln \frac{\exp \left(2 m / r^{s *}\right)-1}{2 m / r^{{ }^{*}}}$, taking derivatives of $f\left(r^{{ }^{*}}\right)$ respecting to $k, a, b, R$ and $\rho$, we have

$$
\begin{aligned}
& \frac{\left.4 k m B-k \rho \bar{t}^{2}+b^{2} \rho B\right) \bar{t}^{2}+4 m b^{2} B^{2}}{2 b B \bar{t}}+2 \\
& \frac{\partial f\left(r^{s *}\right)}{\partial k}=-2 m^{2} b \frac{\frac{2 \sqrt{m\left(4 k m B-\rho\left(k \bar{t}^{2}-b^{2} B\right)\right)}}{\left(b \sqrt{m\left(4 k m R-\rho\left(k \bar{t}^{2}-b^{2} B\right)\right)}+2 k m \bar{t}\right)^{2}}}{2 b}<0 \text {, }
\end{aligned}
$$

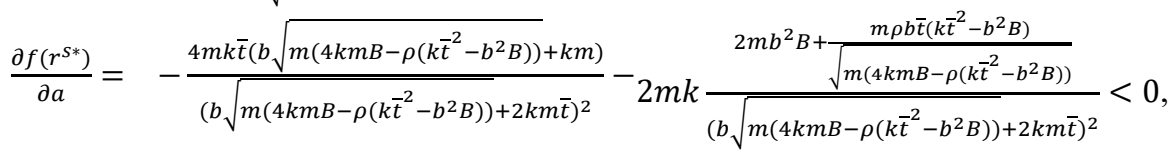

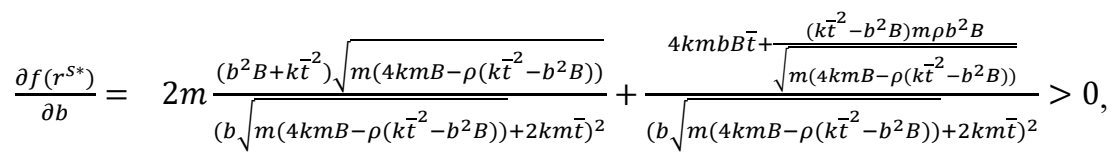

$$
\begin{aligned}
& \frac{\partial f\left(r^{S *}\right)}{\partial B}=\frac{2 m b^{3} \sqrt{m\left(4 k m B-\rho\left(k \bar{t}^{2}-b^{2} B\right)\right)}+4 k m^{2} b^{2} \bar{t}}{\left(b \sqrt{m\left(4 k m B-\rho\left(k \bar{t}^{2}-b^{2} B\right)\right)}+2 k m \bar{t}\right)^{2}}+\frac{\frac{b m^{2}\left(4 k m+b^{2} \rho\right)\left(k \bar{t}^{2}-b^{2} B\right)}{\sqrt{m\left(4 k m B-\rho\left(k \bar{t}^{2}-b^{2} B\right)\right)}}}{\left(b \sqrt{m\left(4 k m B-\rho\left(k \bar{t}^{2}-b^{2} B\right)\right)}+2 k m \bar{t}\right)^{2}}>0, \\
& \frac{\partial f\left(r^{S *}\right)}{\partial \rho}=-\frac{\frac{m^{2} b \rho\left(k \bar{t}^{2}-b^{2} B\right)\left(k \bar{t}^{2}-b^{2} B\right)}{\sqrt{m\left(4 k m B-\rho\left(k \bar{t}^{2}-b^{2} B\right)\right)}}}{\left(b \sqrt{m\left(4 k m B-\rho\left(k \bar{t}^{2}-b^{2} B\right)\right)}+2 k m \bar{t}\right)^{2}}<0 .
\end{aligned}
$$

We complete the proof of Proposition 3 


\section{Appendix H: Proof of Proposition 4}

From Proposition $2, e^{s *}$ can also be written as

$$
\begin{aligned}
e^{S *}= & \frac{b m \rho(a+m-\tau)\left(k(a+m-\tau)^{2}+b^{2} B\right)}{(2 k m(a+m-\tau))^{2}-b^{2} m\left(4 k m B-\rho\left(k(a+m-\tau)^{2}-b^{2} B\right)\right)}+ \\
& \frac{2 m\left(k(a+m-\tau)^{2}-b^{2} B\right) \sqrt{m\left(4 k m B-\rho\left(k(a+m-\tau)^{2}-b^{2} B\right)\right)}}{(2 k m(a+m-\tau))^{2}-b^{2} m\left(4 k m B-\rho\left(k(a+m-\tau)^{2}-b^{2} B\right)\right)}
\end{aligned}
$$

Furthermore, we divide $e^{c *}$ by $e^{s *}$ and have $e^{c *} / e^{s *}=1$.

From $e^{c *}=e^{s *}$, it is straightforward to verify that $r^{S^{*}}=r^{c *}$

\section{Appendix I: Proof of Proposition 5}

The Lagrangian function is

$$
\begin{aligned}
& L^{a}=v r_{h}+(1-v) r_{l}+\lambda_{6}\left(a-\frac{b^{2} \rho \beta_{h} \bar{t}}{4 k_{h} m+b^{2} \rho \beta_{h}}-m+f\left(r_{h}\right)-\tau\right)+\lambda_{7}\left(a-m+f\left(r_{l}\right)-\tau-\frac{b^{2} \rho \beta_{l} \bar{t}}{4 k_{l} m+b^{2} \rho \beta_{l}}\right)-\lambda_{8}\left(B-\alpha_{h}+\right. \\
& \left.\frac{4 k_{h}^{2} m \rho\left(\beta_{h}-1\right) \bar{t}^{2}}{\left(4 k_{h} m+b^{2} \rho \beta_{h}\right)^{2}}\right)-\lambda_{9}\left(B-\alpha_{l}+\frac{4 k_{l}^{2} m \rho\left(\beta_{l}-1\right) \bar{t}^{2}}{\left(4 k_{l} m+b^{2} \rho \beta_{l}\right)^{2}}\right)-\xi_{2}\left(\alpha_{h}+\frac{k_{h} \rho \bar{t}^{2} \beta_{l}}{4 k_{h} m+b^{2} \rho \beta_{l}}-\frac{k_{h} \rho \bar{t}^{2} \beta_{h}}{4 k_{h} m+b^{2} \rho \beta_{h}}-\alpha_{l}\right)-\xi_{1}\left(\alpha_{l}-\frac{k_{l} \rho \bar{t}^{2} \beta_{l}}{4 k_{l} m+b^{2} \rho \beta_{l}}\right) .
\end{aligned}
$$

Taking the derivative of $L^{a}$ with respect to $r_{h}, r_{l}, \alpha_{h}, \alpha_{l}, \beta_{h}$ and $\beta_{l}$, we have

$\frac{\partial L^{a}}{\partial r_{h}}=v+\lambda_{6} f^{\prime}\left(r_{h}\right)=0$,

$\frac{\partial L^{a}}{\partial r_{l}}=1-v+\lambda_{7} f^{\prime}\left(r_{l}\right)=0$,

$\frac{\partial L^{a}}{\partial \alpha_{h}}=\lambda_{8}-\xi_{2}=0$

$\frac{\partial L^{a}}{\partial \alpha_{l}}=\lambda_{9}-\xi_{1}+\xi_{2}=0$,

$\frac{\partial L^{a}}{\partial \beta_{h}}=-\lambda_{6} b^{2} \rho-\lambda_{8} k_{h} \rho \bar{t} \frac{4 k_{h} m-b^{2} \rho \beta_{h}+2 b^{2} \rho}{4 k_{h} m+b^{2} \rho \beta_{h}}+\xi_{2} k_{h} \rho \bar{t}=-\lambda_{6}+\frac{2 \lambda_{8} \bar{t} k_{h} \rho\left(\beta_{h}-1\right)}{4 k_{h} m+b^{2} \rho \beta_{h}}=0$,

$\frac{\partial L^{a}}{\partial \beta_{l}}=\lambda_{7} \frac{-b^{2} k_{l}}{\left(4 k_{l} m+b^{2} \rho \beta_{l}\right)^{2}}+\lambda_{8} \bar{t}\left(\frac{k_{l}^{2}}{\left(4 k_{l} m+b^{2} \rho \beta_{l}\right)^{2}}-\frac{k_{h}^{2}}{\left(4 k_{h} m+b^{2} \rho \beta_{l}\right)^{2}}\right)+\frac{2 \lambda_{9} k_{l} \bar{t}^{2} \rho\left(\beta_{l}-1\right)}{\left(4 k_{l} m+b^{2} \rho \beta_{l}\right)^{3}}=0$.

Because $f^{\prime}(r)<0$, we have $\lambda_{6}>0, \lambda_{7}>0$. From $\lambda_{6} \neq 0$, it follows that $\lambda_{8} \neq 0$. Hence, we have $\lambda_{8}=$ $\frac{\lambda_{6}\left(4 k_{h} m+b^{2} \rho \beta_{h}\right)}{2 \bar{t} k_{h} \rho\left(\beta_{h}-1\right)}>0$ that satisfy $\beta_{h}>1$. From $\lambda_{7}, \lambda_{8}>0$, we have $\lambda_{9}>0$ that satisfy $\beta_{l}>1$. Then we obtain the desired results $\mathbf{m}$.

\section{Appendix J: Proof of Proposition 6}

For the high-type 3PL, from $R=B-\Delta \Pi^{t}$, and $\Delta \Pi^{t}>0$, we have $R<B$. Taking the derivative of $\beta_{h}^{a *}$ with respect to $R$, we have $\frac{\partial \beta_{h}^{a *}}{\partial R}=k_{h} m \bar{t} \frac{4 k_{h} b \bar{t}+\frac{4 k_{h}^{2} m \bar{t}^{2}+b^{2}\left(4 k_{h} m R-\rho\left(k_{h} \bar{t}^{2}-b^{2} R\right)\right)}{\sqrt{m\left(4 k_{h} m R-\rho\left(k_{h} \bar{t}^{2}-b^{2} R\right)\right)}}}{b \rho\left(b^{2} R-k_{h} \bar{t}^{2}\right)^{2}}>0$, i.e., $\beta_{h}^{*}$ is increasing in $R$. From Lemma 2, the 3PL's effort decision e increases in the penalty coefficient $\beta$, so the 3PL's effort decision e increases in $R$. From $R<B$, we have $e_{h}^{c *}>e_{h}^{a *}$.

From $r$ is decreasing in e, we have $r_{l}^{c *}=r_{l}^{a *}, r_{h}^{c *}<r_{h}^{a *}$. Also, because $e_{h}^{a *}=e_{l}^{a *}, r_{h}^{a *}=r_{l}^{a *}$, we have $r_{l}^{c *}=r_{l}^{a *}=$ $r_{h}^{a *}>r_{h}^{c *}$. Under the asymmetric information, the expected tardiness risk is $v r_{h}^{a *}+(1-v) r_{l}^{a *}$, it follows that $r_{l}^{c *}=v r_{h}^{a *}+$ $(1-v) r_{l}^{a *}>r_{h}^{c *}$. The proof of $e_{h}^{a *}=e_{l}^{a *}$ is as follows:

Substitute $R=B-\frac{b^{2} \rho^{2}(a+m-\tau)^{2}\left(k_{l}-k_{h}\right) \beta_{l}^{a * 2}}{\left(b^{2} \rho \beta_{l}^{a *}+4 k_{l} m\right)^{2}}$ into $e_{h}^{a *}$.

$\sqrt{m\left(\left(4 k_{h} m+\rho b^{2}\right)\left(B-\frac{b^{2} \rho^{2} \bar{t}^{2}\left(k_{l}-k_{h}\right) \beta_{l}^{a * 2}}{\left(b^{2} \rho \beta_{l}^{a *}+4 k_{l} m\right)^{2}}-\rho k_{h} \bar{t}^{2}\right)\right.}=2 \bar{t} k_{l} m b \rho\left(b\left(4 k_{h} m+b^{2} \rho\right) B-k_{l} \rho b \bar{t}^{2}+\right.$

$\left.2 k_{h} \bar{t} \sqrt{m\left(4 k_{l} m B-\rho\left(k_{l} \bar{t}^{2}-b^{2} B\right)\right)}\right)$,

$B\left(b^{2} \rho \beta_{l}^{a *}+4 k_{l} m\right)^{2}-b^{2} \rho^{2} \bar{t}^{2}\left(k_{l}-k_{h}\right) \beta_{l}^{a * 2}=4 k_{l}^{2} m \bar{t}^{2} b^{2} \rho^{2}\left(4 m k_{l} k_{h} \bar{t}^{2} B+4 m k_{h} b^{2} B^{2}-\rho\left(B b^{2}-\left(k_{l}-k_{h}\right) \bar{t}^{2}\right)\left(k_{l} \bar{t}^{2}-\right.\right.$ $\left.b^{2} B\right)+4 b k_{h} \bar{t} B \sqrt{\left.m\left(4 k_{l} m B-\rho\left(k_{l} \bar{t}^{2}-b^{2} B\right)\right)\right)}$,

$\left(b^{2} \rho \beta_{l}^{a *}+4 k_{l} m\right)^{2}=4 \bar{t}^{2} k_{l}^{2} m\left(\left(b^{2}\left(4 k_{l} m B-\rho\left(k_{l} \bar{t}^{2}-b^{2} B\right)\right)+4 k_{l}^{2} m \bar{t}^{2}+4 b \bar{t} k_{l} \sqrt{\left.m\left(4 k_{l} m B-\rho\left(k_{l} \bar{t}^{2}-b^{2} B\right)\right)\right) /}\right.\right.$

$\left(k_{l} \bar{t}^{2}-b^{2} B\right)^{2}$, 
$b^{2} \rho \beta_{l}^{a *}+4 k_{l} m=2 \bar{t} k_{l} \frac{2 k_{l} m \bar{t}+b \sqrt{m\left(4 k_{l} m B-\rho\left(k_{l} \bar{t}^{2}-b^{2} B\right)\right)}}{k_{l} \bar{t}^{2}-b^{2} B}$,

After make some basic arithmetic operations of $e_{h}^{a *}$, we can derive that $e_{h}^{a *}=e_{l}^{a *}$. Thus, we complete the proof of Proposition 6 .

Appendix K: Proof of Proposition 7

From the Proof of the Proposition 6, we know that $\beta$ is increasing in $R$, since $R<B$, we have $\beta_{h}^{a *}<\beta_{h}^{s *}$. Taking derivatives of $\alpha_{h}^{a *}$ with respect to $\beta_{h}^{a *}$, we have $\frac{\partial \alpha_{h}^{a *}}{\partial \beta_{h}^{a *}}=4 k_{h}^{2} m \rho(a+m-\tau)^{2} \frac{4 k_{h} m+2 b^{2} \rho-b^{2} \rho \beta_{h}^{a *}}{\left(b^{2} \rho \beta_{h}^{a *}+4 k_{h} m\right)^{3}}$, then we have the following two scenarios. 1) If $\beta_{h}^{a *}<\frac{4 k m}{b^{2} \rho}+2$, we have $\frac{\partial \alpha_{h}^{a *}}{\partial \beta_{h}^{a *}}>0$,i.e., $\alpha_{h}^{a *}<\alpha_{h}^{S *}$; 2) If $\beta_{h}^{a *}>\frac{4 k m}{b^{2} \rho}+2$, we have $\frac{\partial \alpha_{h}^{a *}}{\partial \beta_{h}^{a *}}<0$, i.e., $\alpha_{h}^{a *}>\alpha_{h}^{S *}$. Because $\beta_{l}^{a *}<$ $\beta_{l}^{s *}$, it follows that $\alpha_{l}^{a *}=\alpha_{l}^{S *}$. Thus, we complete the proof of Proposition $7 \mathbf{m}$.

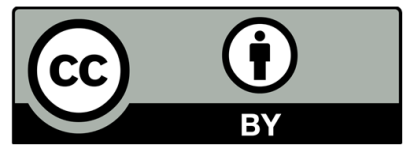

(C) 2022 by the authors; licensee Growing Science, Canada. This is an open access article distributed under the terms and conditions of the Creative Commons Attribution (CCBY) license (http://creativecommons.org/licenses/by/4.0/). 